\title{
Liquid-Based Cytology of the Cerebrospinal Fluid in a Case of Cryptococcal Meningitis
}

\author{
Jiwoon Choi · Se Hoon Kim ${ }^{1}$ \\ Department of Pathology, Yonsei University \\ Wonju College of Medicine, Wonju; \\ ${ }^{1}$ Department of Pathology, Yonsei University \\ College of Medicine, Seoul, Korea \\ Received: April 30, 2017 \\ Revised: June 11, 2017 \\ Accepted: June 13, 2017

\section{Corresponding Author} \\ Se Hoon Kim, MD, PhD, FIAC, EFN \\ Department of Pathology, Yonsei University College \\ of Medicine, 50-1 Yonsei-ro, Seodaemun-gu, Seoul \\ 03722, Korea \\ Tel: +82-2-2228-1769 \\ Fax: +82-2-362-0860 \\ E-mail: paxco@yuhs.ac
}

\begin{abstract}
Cryptococcus neoformans is the most common microorganism found in cerebrospinal fluid (CSF) cytology and causes life-threatening infections in immunocompromised hosts. Although its cytomorphologic features in conventional smear cytology have been well described, those in liquidbased cytology have rarely been. A 73-year-old woman with diffuse large B-cell lymphoma presented with mental confusion and a spiking fever. To rule out infectious conditions, CSF examination was performed. A cytology slide that was prepared using the ThinPrep method showed numerous spherical yeast-form organisms with diameters of 4-11 $\mu \mathrm{m}$ and thick capsules. Occasional asymmetrical, narrow-based budding but no true hyphae or pseudohyphae were observed. Gomori methenamine silver staining was positive. Cryptococcosis was confirmed in blood and CSF through the cryptococcal antigen test and culture. Liquid-based cytology allows for a clean background and additional slides for ancillary testing, facilitating the detection of microorganisms in CSF specimens, particularly when the number of organisms is small.
\end{abstract}

Key Words: Cryptococcus neoformans; Cerebrospinal fluid; Liquid-based cytology
Cryptococcus neoformans is the most common microorganism found in cerebrospinal fluid (CSF) cytology. ${ }^{1}$ Detecting C. neoformans in CSF specimens is crucial for cytopathologists as the organism causes life-threatening infections in immunocompromised hosts. Although the cytomorphologic features of $C$. neoformans in conventional smear cytology have been well described, those in liquid-based cytology have rarely been. Recently, a case in sputum cytology sample has been reported. ${ }^{2}$ Here, we report the findings of a liquid-based cytologic preparation of a CSF specimen in a case of culture-proven cryptococcal meningitis.

\section{CASE REPORT}

A 73-year-old woman visited the hospital with complaints of myalgia, night sweats, and anorexia for 1 week. Her past medical history was unremarkable. On physical examination, a palpable mass was discovered in the right neck, and abdominopelvic computed tomography showed generalized lymphadenopathy. Excisional biopsy of the cervical mass revealed diffuse large Bcell lymphoma, and she received chemotherapy treatment. After completing 1 cycle of chemotherapy, she presented with a spiking fever (up to $38.3^{\circ} \mathrm{C}$ ) and mental confusion. Laboratory tests revealed leukocytosis (white blood cell count, $11.01 \times 10^{3} / \mu \mathrm{L}$ ) and increased C-reactive protein levels $(207.3 \mathrm{mg} / \mathrm{L})$ in peripheral blood. Therefore, CSF examination by lumbar puncture was performed to rule out infectious meningitis.

The CSF specimen submitted for cytologic examination was clear and colorless. It was processed onto a ThinPrep slide and stained with the Papanicolaou stain. Microscopic examination revealed many round to oval structures with thick halos (Fig. 1). They stained pale blue and ranged in size from 4 to $11 \mu \mathrm{m}$. The initial impression was a contaminant, such as glove powder, because the structures were numerous while the background was almost acellular (Fig. 1A). However, when viewed carefully, the structures contained a brownish dot-like internal structure that was suspected as being a nucleus, with occasional tear drop-shaped budding (Fig. 1B, C). When an additional slide was stained with the Gomori methenamine silver method, they stained black (Fig. 1D). Based on positive cryptococcal antigen detection and India ink tests of the CSF specimen prior to the initial cytologic diagnosis, the presence of $C$. neoformans was reported. Subsequently, CSF and blood cultures confirmed cryptococcosis. Although treatment with amphotericin B was started and the organisms were not seen in a repeat CSF examination, the patient died approximately 3 


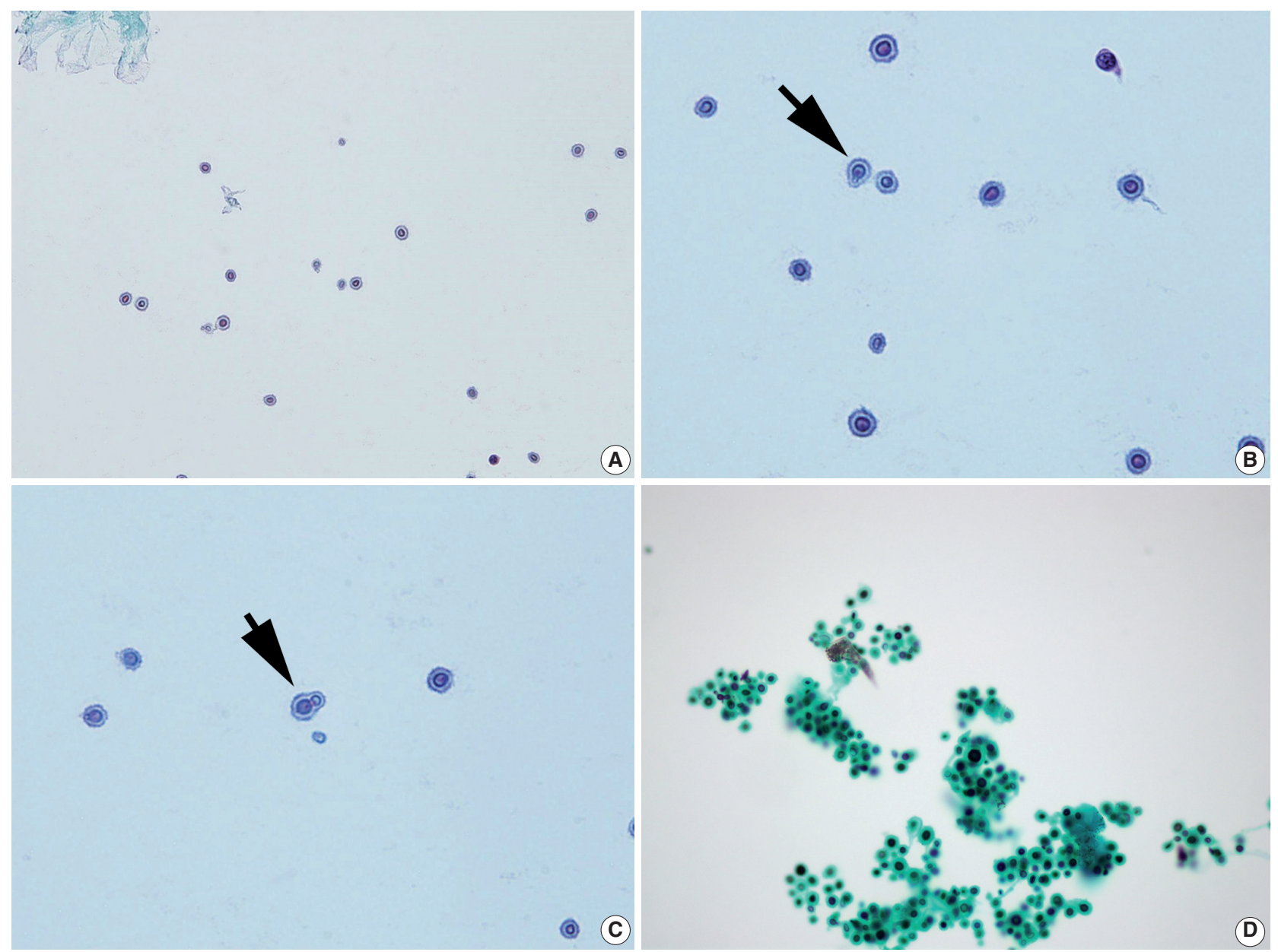

Fig. 1. Representative images of ThinPrep slide preparations of the cerebrospinal fluid. (A) Liquid-based cytology shows numerous spherical yeast cells against a clean background (Papanicolau stain). (B, C) The cells have varying sizes, clear halos, and show occasional narrowbased single budding (arrows) (Papanicolau stain). (D) Black staining is observed with the Gomori methenamine silver stain.

months later owing to worsening of her general condition and recurrence of cryptococcal meningitis.

The authors comply with the guidelines for human studies. The patient died approximately 3 months later owing to worsening of her general condition and recurrence of cryptococcal meningitis. Therefore, the authors could not receive the patient's informed consent for this manuscript. This was notified to the Institutional Review Board (IRB), who waived the IRB protocol approval (4-2017-0814).

\section{DISCUSSION}

C. neoformans is a ubiquitous encapsulated yeast that causes infections ranging from asymptomatic pulmonary colonization to life-threatening meningoencephalitis. ${ }^{3} \mathrm{C}$. neoformans enters the body by inhalation through the respiratory tract and spreads hematogenously to the central nervous system. It has long been recognized that while $C$. neoformans may cause meningoencephalitis in otherwise healthy people, it more frequently presents as an opportunistic infection in immunocompromised individuals with acquired immunodeficiency syndrome, neoplastic conditions, or those undergoing corticosteroid therapy; it is fatal without treatment. Therefore, rapid recognition and diagnosis are required to decrease mortality rates.

The cytomorphology of $C$. neoformans in conventional smear cytology has been well described. ${ }^{4,5}$ Cryptococcus has yeast but not pseudohyphal or hyphal forms with rare exceptions. With the routine Papanicolaou stain, yeast cells can be seen as pale, brownish-pink, slightly refractile spheres surrounded by clear halos. In Diff-Quik-stained preparations, purple yeast cells with accentuated clear halos against a dark purple background give the smear a punched-out appearance. Cells generally range from 
5 to $10 \mu \mathrm{m}$ in diameter, but this can vary from 2 to $20 \mu \mathrm{m}$. A useful cytologic feature is the appearance of the asymmetric, narrow-based budding. Special stains, such as mucicarmine, periodic acid-Schiff and alcian blue, can aid the diagnosis as Cryptococcus has a characteristic thick mucopolysaccharide capsule. In patients with cryptococcal meningitis, the inflammatory response shows variable degrees depending on the immunocompetence of the hosts. ${ }^{6}$ While CSF smears from immunocompetent patients typically manifest as CSF pleocytosis, smears from immunocompromised patients often show large numbers of yeast cells without an inflammatory response, as shown in our case.

Recently, Cryptococcus gatti, which is closely related to C. neeformans, has emerged in North America and has been responsible for a large disease epidemic in a population of generally healthy individuals including immunocompromised hosts. ${ }^{7}$ Unfortunately, it is difficult to differentiate $C$. gatti from C. neoformans by morphology alone. Further differentiation of cryptococcal species can be accomplished on L-canavanine-glycine-bromthymol blue agar; $C$. gattii colours the medium blue while $C$. neoformans does not (the medium remains yellow). ${ }^{8}$

In the cytologic examination of non-gynecological body fluids, including CSF, the liquid-based preparation method has not been used as widely as the conventional method. Moreover, the literature describing the morphologic features of cryptococci in liquid-based cytology is scarce. In this report, we observed that the most important cytomorphologic features of cryptococci, such as the thick capsule and tear-drop shaped budding, are well maintained in liquid-based cytology. Moreover, liquid-based cytology provided a clean background that facilitated the detection of the microorganisms and allowed for the preparation of additional slides for special staining to confirm the diagnosis.

In patients with cryptococcal meningitis, cytologic examination of the CSF has been shown to have a low diagnostic yield as the yeast cells can be easily overlooked, particularly when few in number, and can be confused with erythrocytes or artifacts (e.g., surgical glove powder, dust, or glass powder). ${ }^{4}$ Moreover, cryptococci sometimes show unusual cytomorphology that can cause diagnostic difficulty, such as chains of budding yeasts, pseudohyphae, or capsule-deficient forms. ${ }^{10,11}$ Our experience in this case suggests that liquid-based cytology may improve the sensitivity of the detection of microorganisms and thus diagnostic accuracy by providing opportunities for ancillary testing. In addition, for CSF examinations, liquid-based cytology might be superior to conventional cytology for clear morphology, as cellular components can be deformed in the latter during cytospin preparations.

\section{Conflicts of Interest}

No potential conflict of interest relevant to this article was reported.

\section{REFERENCES}

1. Prayson RA, Fischler DF. Cerebrospinal fluid cytology: an 11-year experience with 5951 specimens. Arch Pathol Lab Med 1998; 122: 47-51.

2. Sharma S, Gupta N, Behera D, Rajwanshi A. Detection of cryptococcosis in liquid-based sputum cytology. Cytopathology 2017; 28: $177-8$

3. Levitz SM. The ecology of Cryptococcus neoformans and the epidemiology of cryptococcosis. Rev Infect Dis 1991; 13: 1163-9.

4. Saigo P, Rosen PP, Kaplan MH, Solan G, Melamed MR. Identification of Cryptococcus neoformans in cytologic preparations of cerebrospinal fluid. Am J Clin Pathol 1977; 67: 141-5.

5. Powers CN. Diagnosis of infectious diseases: a cytopathologist's perspective. Clin Microbiol Rev 1998; 11: 341-65.

6. Walts AE. Cerebrospinal fluid cytology: selected issues. Diagn Cytopathol 1992; 8: 394-408.

7. Dixit A, Carroll SF, Qureshi ST. Cryptococcus gattii: an emerging cause of fungal disease in North America. Interdiscip Perspect Infect Dis 2009; 2009: 840452.

8. Kwon-Chung KJ, Polacheck I, Bennett JE. Improved diagnostic medium for separation of Cryptococcus neoformans var. neoformans (serotypes A and D) and Cryptococcus neoformans var. gattii (serotypes B and C). J Clin Microbiol 1982; 15: 535-7.

9. Kobayashi TK, Ueda M, Nishino T, Moritani S, Higaki T, Bamba M. Cytologic detection of cryptococcosis coexisting with herpes simplex virus infection in sputum: use of liquid-based, thin-layer preparations. Acta Cytol 2003; 47: 103-6.

10. Williamson JD, Silverman JF, Mallak CT, Christie JD. Atypical cytomorphologic appearance of Cryptococcus neoformans: a report of five cases. Acta Cytol 1996; 40: 363-70.

11. Kanazawa M, Ishii M, Sato Y, Kitamura K, Oshiro H, Inayama Y Capsule-deficient meningeal cryptococcosis. Acta Cytol 2008; 52: 266-8. 